\title{
Nasopalatine Cyst: A Silent Growing Lesion
}

\author{
Ángel Terrero-Pérez, Mariela Peralta-Mamani, Eduardo Sant'Ana, José Humberto Damante, Alberto \\ Consolaro, Izabel Regina Fischer Rubira-Bullen and Ana Lúcia Alvares Capelozza*
}

Department of Stomatology, Bauru School of Dentistry, University of São Paulo, Brazil

Received: August 18, 2017; Published: September 06, 2017

*Corresponding author : Ana Lúcia Alvares Capelozza, Associate Professor at the Bauru School of Dentistry, University of São Paulo (FOB-USP), Department of Stomatology, Alameda Octávio Pinheiro Brisola, 9-75, Vila Universitária, 17012-901 Bauru, SP, Brazil, Tel: 5514998 308803; Email: anacapel@fob.usp.br

\section{Abstract}

The nasopalatine cyst (NPC) is a non-odontogenic cyst, it occurs mainly in adult patients in some cases can bring difficulties to the diagnostic due to its silent growing. We are going to present in this paper report case in a 10-year child emphasizing the histophatological characteristics and the findings in obtained images by Cone Beam Computed Tomography (CBCT).

Keywords: Non-odontogenic cysts; Nasopalatine cyst; Image diagnostic

\section{Introduction}

The nasopalatine cyst (NPC) represents from 38 to $43.66 \%$ of the non-odontogenic cysts, followed by oral lympho epithelial cyst and epidermoid cyst. This cyst wound still represents about 1.87 to $10 \%$ of the jaw cysts. With major frequency in men, between the fourth and sixth decades [1-3].This non-odontogenic cyst is labeled as a development cyst, and its pathogenesis are not clear described yet, are believed to arise from the remnants of nasopalatine ducts in the incisive canal [4]

For the most part are asymptomatic, sometimes NPCs are oligosymptomatic, slow growth and discovered in image exams done for other purposes. Symptomatic in about $48 \%$ of the patients [5], in signs and irritation presence [6]. The patients present as frequent main complain the "swelling" well defined associated to pain by secondary irritation or nasopalatine nerve pressure, therefore the most common symptom is the tumefaction usually placed in the front region of the palate medium line. It can be present in the medium line of the vestibular region in the alveolar ridge and in some cases, vestibular and palate tumefaction [4] The bone expansion, abscess, slow healing after dental extraction, bulging in the nasal cavity and distortion of the nasal septum are other found signs $[7,8]$. The cystic content can overflow and run down to the oral cavity, in some cases, the reason of the patient report of salty taste in the mouth described by some patients $[2,8]$.

The symptoms described by the patients are not related to the size of the cyst. However, the dimensions present correlation with the post-surgery complications in 32 to $53 \%$ of the cases. Amongst the post-surgery complications are infections, devitalized central incisors, fistula and paresthesia placed in the palate $[5,8]$. The conventional radiographic image shows the expanded incisive foramen with definite edges, oval or round shape, when the nose spine overlaps itself, a radiolucent heart-shape image may be observed as well as deformation of the lateral and anterior limit of the nasal floor $[2,6]$. Clinically, can be shown as clear visible ridges beside the incisive papilla even as a fistula that extends itself from the mouth to the nasal cavity. Through the catheterization maneuver of the persistent and total or part latent nasopalatine duct, with gutta-percha cone and periapical radiography, it allows the visualization of the lumen passage [9-10]. Besides, it can cause displacement and adjacent structures, expansion of the cortical vestibular bone and palatine, swelling of the incisive foramen and upper displacement of the nasal cavity floor $[2,6,11]$. The diagnostic depends on clinical examination, diagnostic imaging and histophatological. The recommended treatment is the enucleation of the lesion [7].

\section{Case Report}

A leukoderma 10-years female patient, student was submitted to a panoramic exam due orthodontics purposes. An as incidental finding, an extended radiolucent intraosseous lesion in the anterior maxilla was observed. Although the lesion was very extensive, no symptoms were reported, and the patient had no idea of the lesion until the panoramic radiography was done. Thus, was conducted to our service, after anamnesis and physical examination, a Cone Beam Computed Tomography (СBCT) was requested, with a field of view of $6 \times 6 \mathrm{~cm}$ to better evaluation of the interested region (I-CAT, Imaging Science International).

The CBCT image revealed a remarkable hypodense lesion, well delimited, with approximately $2.2 \times 3.5 \mathrm{~cm}$ in diameter, in the palate from the incisors to first pre-molars, expanding the vestibular 
cortical, palate and the floor of the nasal cavity. As an incidental finding, an image compatible with the mucous retention cyst in the maxillary sinus on left side and thickening of the mucosa of the sinus right maxillary sinus was also observed. The roots of adjacent teeth were intact, with a slight protrusion of the tooth 21 and slight displacement of the roots of the teeth 11 and 21 (Figures 1-3). In the medical history, the father reported that the patient has undergone surgery for adenoidectomy; it was detected mouth breathing in the patient.

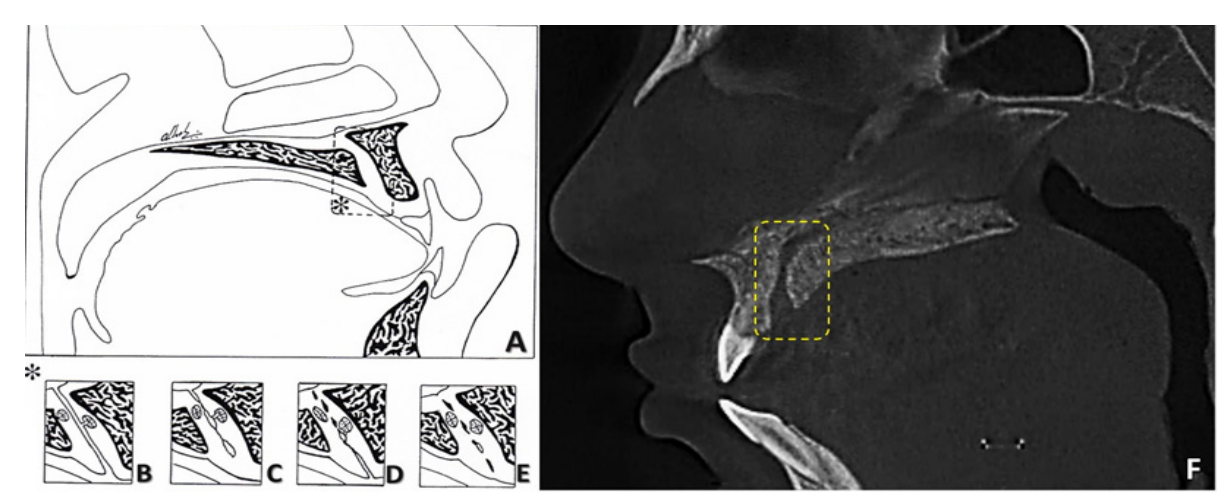

Figure 1: (A-E) Schematic figures of the persistence of the nasopalatine duct, and their various forms. (A) Anatomical aspects of the nasopalatine canal, and its relationships with adjacent tissues in a sagittal view, in the highlighted area $\left({ }^{*}\right)$ there are four possibilities of persistence of the structure and / or remnants of the nasopalatine duct. (B) Persistent nasopalatine duct in its total patent form. (C) Partial patent nasopalatine duct with opening facing to the nasal cavity. (D) Partial patent nasopalatine duct with oral opening. (E) Remnants of the nasopalatine duct in the form of epithelial cords, eventually presenting on the inside of the duct. (F) Sagital reconstruction of TCFC show a normal incisive canal, without nasopalatine cyst.

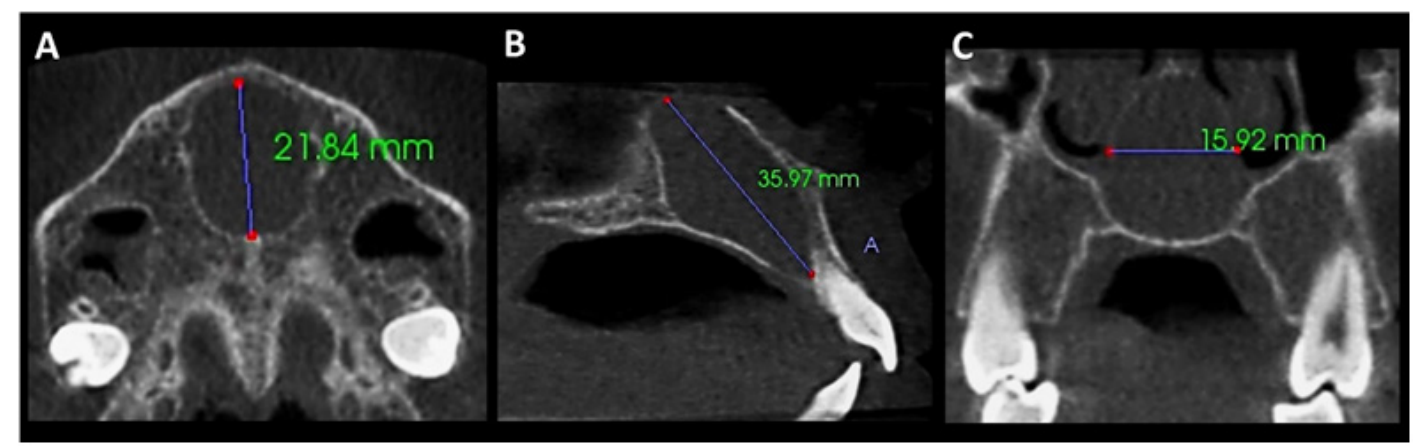

Figure 2: (A, B and C) Axial, sagittal and coronal reconstructions slices showing the dimensions of the nasopalatine cyst.

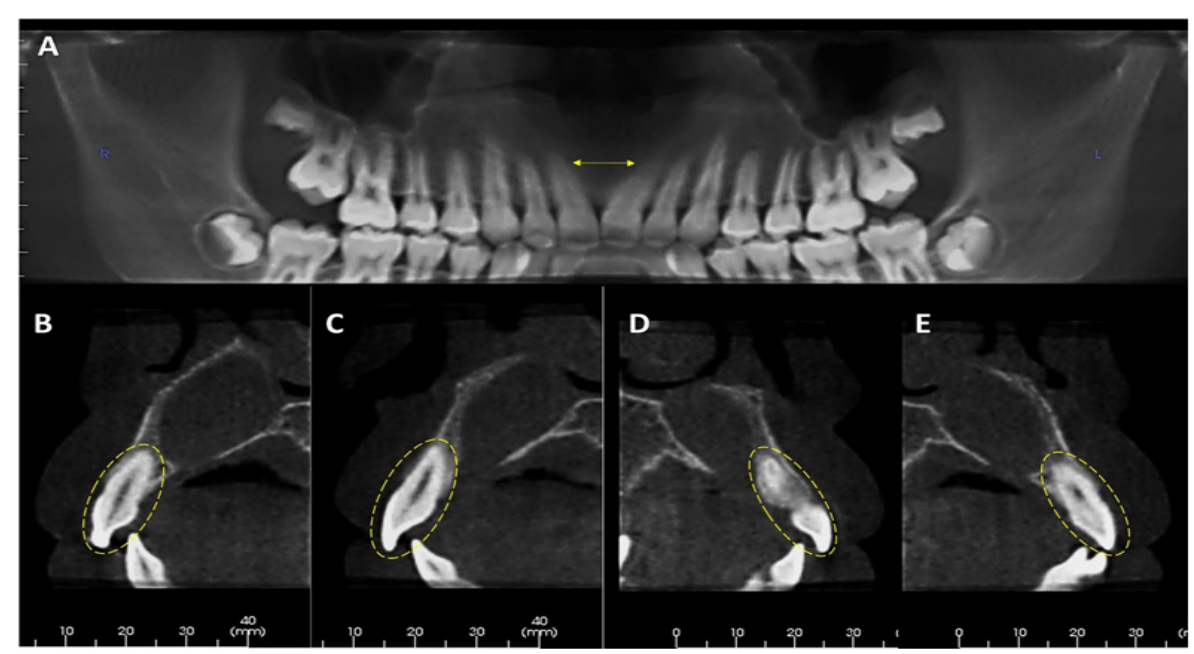

Figure 3: (A) Panoramic reconstruction shows slight displacement of the roots of the teeth 11 and 21, indicated by the arrows. (B-F) Parasagittal reconstructions of the teeth 12,11, 21 and 22, respectively, show the absence of external root resorption. 
After clinical evaluation and the images, the presumptive diagnosis was of NPC, the patient underwent surgery under general anesthesia in the Hospital for Rehabilitation of Craniofacial Anomalies-University of São Paulo (HRAC-USP) for removal of the lesion. In the fine-needle aspiration was detected the presence of liquid dark brown and therefore it was performed a surgical enucleation of the lesion. The fragment removed was sent to histopathological examination, the macroscopical showed a soft tissue of elastic consistency, shape and surface of irregular shape, blackened color, measuring 1.3 x 1.2 × $0.6 \mathrm{~cm}$ (Figures 4-6).

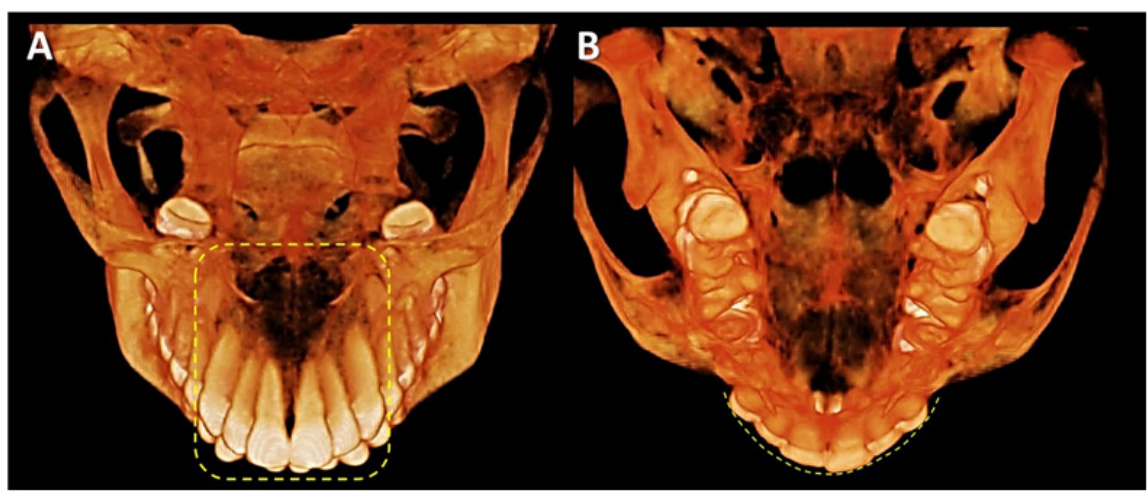

Figure $4:$ (A and B) 3D reconstructions. (A) Highlighted area shows integrity of the roots of adjacent teeth to the nasopalatine cyst. (B) Image show slight protrusion of the tooth 21.

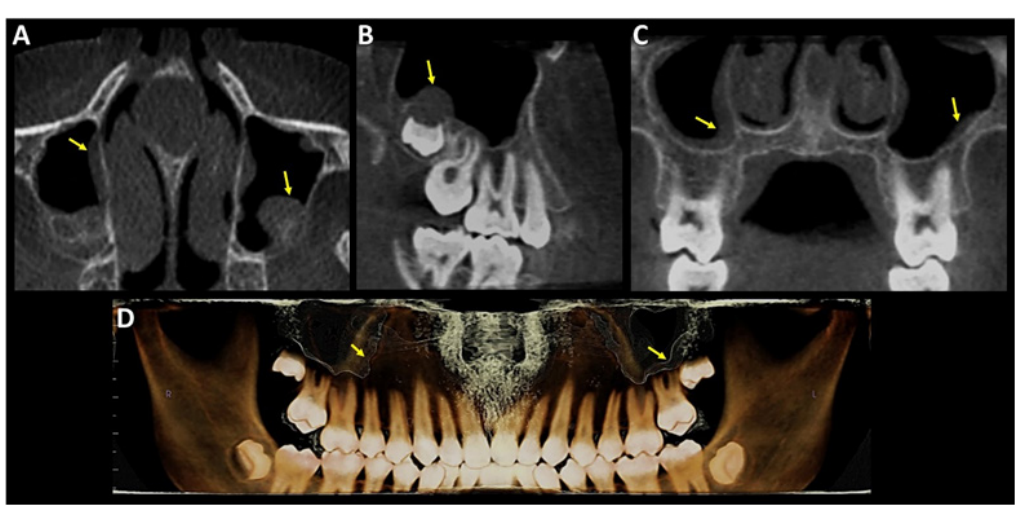

Figure 5 : Axial, sagittal, coronal and 3D reconstructions slices, respectively, showing bilateral maxillary sinus mucosal thickening, indicated by the arrows.
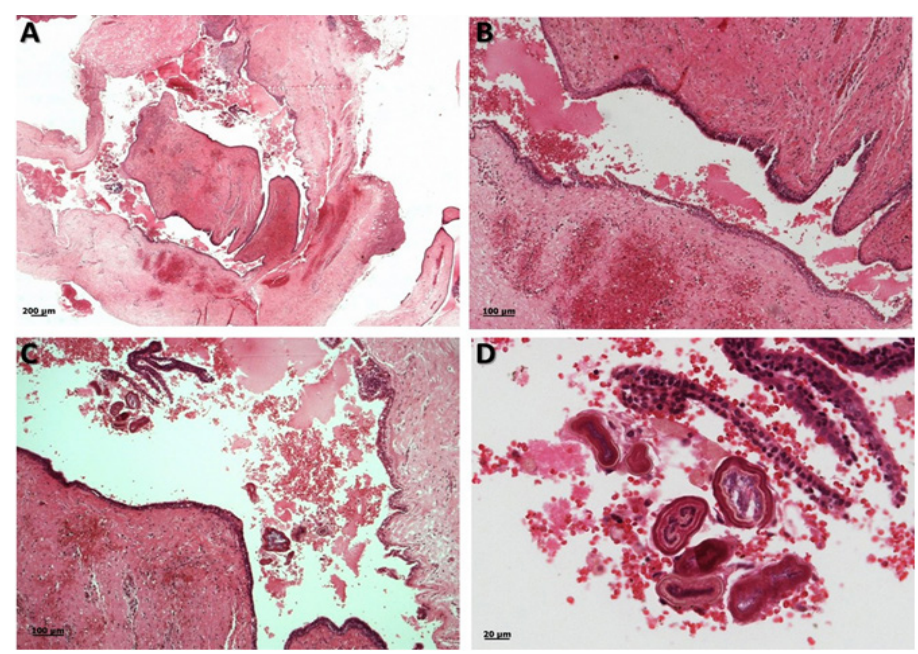

Figure 6 : The microscopic images revealed (A, B) fibrous connective tissue in the shape with virtual cystic cavity coated predominantly by the pseudostratified ciliated epithelium and stratified pavement epithelium, also there are blood vessels caliber, neural structures and fatty tissue, still, mononuclear inflammatory cells diffusely distributed (C, D). (H.E. Original increase: $A=4 x$; $B$ and $C=10 x$ and $D=25 x$ ). 
The microscopic examination revealed a virtual cystic cavity coated predominantly by the pseudostratified ciliated epithelium. The fibrous capsule behind presented many arteries and veins, as well as, nervous bundles thick and fatty tissue, and may even observe mononuclear inflammatory cells distributed in such a way as diffuse, extensive hemorrhagic areas and bone fragments (Figure 2). The histopathological diagnosis was conclusive of nasopalatine cyst, as the diagnosis hypothesis. In the postoperative follow-up there were no clinical or radiographic signs of lesion recurrence.

\section{Discussion}

The diagnosis made by the dental surgeon depends on the information obtained from the patient during anamnesis, clinical examination, and in some cases it is necessary to choose the requisition of complementary examinations, such as the histopathological and imaging exams. Amongst the imaging exams available are the images in 2D known as conventional (intra and extra oral) and the 3D images obtained using dental tomographs (CBTC), which assists a lot to evaluation of structures and helps the dental surgeon with important information, often essential in the correct diagnosis and surgical planning. Particularly those extensive lesions and asymptomatic that involve the anatomical structures of the maxilla and mandible.

The incisive canal and the nasopalatine duct (NPD) are two structures anatomically different. The incisive canals pass through on each side of the nasal septum to extend from the nasal cavity to the oral cavity, already on the path to the oral cavity, these channels join and leave in an opening in the common area of the incisive papilla. The NPD is an organ that humans have only remnants of epithelium within the incisive canal, which can give rise to the nasopalatine cyst (NPC) [12-13].

The frequency of the NPC was reported by Muzio et al. [3] in $86.7 \%$ of the non-odontogenic cysts in a sample with 30 cases and $1.5 \%$, among all the cystic lesions of the jaws of 1716 cases. Higher in men in a ratio 5.5:1 compared to cases presented in women, the average age of occurrence was 47.9 years. Another study also showed a frequency lower in women [8]. Our report is of a 10-yearold female child, an uncommon finding. Regarding to the location of the NPC, the higher volume and frequency is in the front region more below, may explain why most of the NPC are central to that NPC unilateral or bilateral [13]. Coinciding with the case report presented, where the lesion was located median region of the maxilla.

Faitaroni et al. [14] described two clinical cases of NPC with initial diagnosis of periapical lesions, reported that the images of CBCT helped in the diagnosis and surgical planning of NPC and that the histopathological examination remains as the standard required in the establishment of the final diagnosis of lesions of the jaws. The present report is similar to that described by these authors in relation to the surgical procedure, surgical planning was carried out by means of CBCT, as well as also confirmed the diagnosis by means of histopathological examination.

In the case reported, the NPC presented asymptomatic, even though the lesion was extensive in the median region of maxilla, however, may manifest with symptoms as was shown by Suter et al., [8] with up to $65 \%$ of cases, being the most frequent, the swelling associated with abscess with $17.5 \%$, pressure with $12.5 \%$ and pain with $12.5 \%$. The diameter of the NPC reports submitted was on average $2.85 \mathrm{~cm}$, by In Vivo software, uncommon, some studies describe how average size $1.5 \mathrm{~cm}$ in diameter. The men showed an average size of $1.4 \mathrm{~cm}$ and the women of $1.2 \mathrm{~cm}$ in diameter [8]. When the expansion of the incisive foramen is discreet, it could make diagnosis difficult, therefore, images larger than $10 \mathrm{~mm}$ should be proserved.

In the differential diagnosis, it is important to exclude the possibility of a lesion periapical, as the apical periodontal cyst, testing the pulp vitality of the upper incisors [4]. Therefore, in endodontics is important to make this determination to avoid performing treatment of canal in teeth with pulp vitality, as in the case of the presence of the NPC, the canal treatment would not solve the signs and symptoms of the patient and only the enucleation of the lesion. Furthemore, it has to be differentiated from the odontogenic keratocyst of the anterior region of the maxilla, particularly in cases in with vital central incisors. Thus, the pulp vitality of the upper incisors is essential for NPC diagnosis.

\section{Conclusion}

The exams obtained in dental tomographs that allow us to view the image in 3D are very effective, in not only the presumptive diagnosis, but also especially in safety during the surgical procedure, reducing your time and improving postoperative period. The silent growing of the NPC may occur without any acknowledge for the patient, therefore, despite being a benign cyst, NPC can achieve large size leading to complications or deformities trans and post surgical treatment. The final diagnosis should be made with the addition of clinical information, imaging, histopathological and surgical results.

\section{References}

1. Pode D, Meretik S, Shapiro A (1985) Diagnosis and management of renal angiomyolipoma. Urology 25: 461-467.

2. Srinivasan V, Turner AG, Blackford HN (1994) Massive intraperitoneal hemorrhage associated with renal pathology. J Urol 151(4): 980-981.

3. Hayman J, Leiter E (1987) Spontaneous retroperitoneal hemorrhage: unusual presentation of renal cell cancer. Urology 30: 259-261.

4. Spires AM, Gaede JT, Glenn JF (1980) Death from renal cyst. Urology 16: 606-607.

5. Buller HR, Agnelli G, Hull RD (2004) Antithrombotic therapy for venous thromboembolic disease: the Seventh ACCP Conference on Antithrombotic and Thrombolytic Therapy. Chest 126(3): 401S-428S.

6. Topgul K, Uzun O, Anadol AZ, Gok A (2005) Surgical management of enoxaparin-and /or warfarin-induced massive retroperitoneal bleeding: report of a case and review of the literature. South Med J 98(1): 104-106.

7. Mrug M, Mishra PV, Lusane HC, Cunningham JM, Alpert MA (2002) Hemothorax and retroperitoneal hematoma after anticoagulation with enoxaparin. South Med J 95(8): 936-938.

8. Chan-Tak KM (2003) Fatal retroperitoneal hematoma secondary to enoxaparin. South Med J 96: 58-60.

9. Daliakopoulos SI, Bairaktaris A, Papadimitriou D, Pappas P (2008) Gigantic retroperitoneal hematoma as a complication of anticoagulation therapy with heparin in therapeutic doses: a case report. J Mexd Case Reports 2: 162. 
10. Belville JS, Morgentaler A, Loughlin KR, Tumeh SS (1989) Spontaneous perinephric and subcapsular renal hemorrhage: Evaluation with CT, US, and angiography. Radiology 172(3): 733-738.

11. Sherer DM, Dayal AK, Schwartz BM, Oren R, Abufalia O (1999) Extensive spontaneous retroperitoneal hemorrhage: an unusual complication of heparin anticoagulation during pregnancy. J Matern Fetal Med 8: 196199.

12.Amano T, Takemae K, Niikura S, Kouno M, Amano M (1999) Retroperitoneal hemorrhage due to spontaneous rupture of adrenal myelolipoma. Int J Urol 6(11): 585-588.

\begin{tabular}{|l}
\hline BIOMEDICAL \\
RESEARCHES
\end{tabular}$\quad$\begin{tabular}{l} 
Assets of Publishing with us \\
\hline
\end{tabular}

